\title{
Synthesis of coaxial nanotubes of polyaniline and poly(hydroxyethyl methacrylate) by oxidative/initiated chemical vapor deposition
}

\author{
Alper Balkan $¥ 1$, Efe Armagan $¥ 1$ and Gozde Ozaydin Ince ${ }^{\star 1,2}$
}

\author{
Full Research Paper \\ Address: \\ ${ }^{1}$ Materials Science and Nanoengineering Program, Faculty of \\ Engineering and Natural Sciences, Sabanci University, Tuzla, Istanbul \\ 34956, Turkey and ${ }^{2}$ Nanotechnology Application Center, Sabanci \\ University, Orhanli, Tuzla, Istanbul 34956, Turkey \\ Email: \\ Gozde Ozaydin Ince* - gozdeince@sabanciuniv.edu \\ * Corresponding author $\ddagger$ Equal contributors \\ Keywords: \\ coaxial nanotubes; humidity sensors; initiated chemical vapor \\ deposition; oxidative chemical vapor deposition; polyaniline
}

Beilstein J. Nanotechnol. 2017, 8, 872-882. doi:10.3762/bjnano.8.89

Received: 15 January 2017

Accepted: 21 March 2017

Published: 18 April 2017

This article is part of the Thematic Series "Vapor-based polymers: from films to nanostructures".

Guest Editor: M. Koenig

(C) 2017 Balkan et al.; licensee Beilstein-Institut. License and terms: see end of document.

\begin{abstract}
Vapor-phase synthesis techniques of polymeric nanostructures offer unique advantages over conventional, solution-based techniques because of their solventless nature. In this work, we report the fabrication of coaxial polymer nanotubes using two different chemical vapor deposition methods. The fabrication process involves the deposition of an outer layer of the conductive polyaniline (PANI) by oxidative chemical vapor deposition, followed by the deposition of the inner layer of poly(2-hydroxyethyl methacrylate) (pHEMA) hydrogel by initiated chemical vapor deposition. The vapor-phase techniques allowed for fine-tuning of the thickness of the individual layers, keeping the functionalities of the polymers intact. The response of the single components and the coaxial nanotubes to changes in humidity was investigated for potential humidity sensor applications. For single-component conductive PANI nanotubes, the resistance changed parabolically with relative humidity because of competing effects of doping and swelling of the PANI polymer under humid conditions. Introducing a hydrogel inner layer increased the overall resistance, and enhanced swelling, which caused the resistance to continuously increase with relative humidity.
\end{abstract}

\section{Introduction}

In recent years, with the advances in nanotechnology, the use of nanostructured materials has become widespread in various applications, such as biotechnology $[1,2]$, food industry $[3,4]$, sensors [5] or photovoltaics [6]. Polymeric nanostructures have attained special interest because of their prominent advantages, such as cost-effectiveness, ease of fabrication and biocompatibility making these nanostructures applicable in different areas $[7,8]$. Among these, the conducting polymer (CP) nanostruc- 
tures, such as nanowires, nanorods, nanotubes or nanospheres have been extensively studied through solution-based techniques, such as chemical polymerization [9-11] or electrochemical polymerization [12-14] for applications in light emitting diodes [15], photovoltaic cells [16,17], supercapacitors [18], sensors [19] and drug delivery [20]. During synthesis of these nanostructures, the use of solvents is a major drawback for homogeneity and conformal coatings, especially on highaspect-ratio templates, due to wetting effect and surface tension. Thus, vapor-phase polymerization techniques have emerged for the deposition of conducting polymers that facilitate the fabrication of conformal polymeric structures [21,22].

Polyaniline (PANI) is one of the well-known conducting polymers with applications in supercapacitors [18], sensors [23], solar cells [24] and membranes [25] because of its great thermal and environmental stability, ease of synthesis, excellent conductivity, cost-effectiveness and redox-tunability [26-29]. One of the original aspects of PANI is that its electrical conductivity can be tuned through oxidation and protonation steps. Depending on the oxidation level, PANI can exist in three different states: leucomeraldine base (fully reduced), emeraldine base (half-oxidized) and pernigraniline base (fully oxidized). However, only the emeraldine salt which is the protonated form of emeraldine has a good conductivity of $1-130 \mathrm{~S} / \mathrm{cm}$ [30,31].

PANI is a good candidate material for sensor applications due to the change of oxidation/reduction level in response to changes in the environmental conditions, which, in turn, affect the electrical conductivity [32]. However, studies on PANI as humidity sensors are very limited. Zeng et al. [33] studied the resistance change of PANI nanofibers depending on the humidity level. They observed that the resistance changed parabolically as the humidity of the environment increased, and a minimum value for the resistance at a certain humidity level existed. The parabolic behavior with the same resistance readings for two different humidity levels is problematic for sensor applications, making the sensor unreliable. In order to modify this parabolic behavior, Parvatikar et al. [34] fabricated PANI/ $\mathrm{CeO}_{2}$ composites, whose resistance values decreased linearly as humidity increased due to charge transfer between $\mathrm{CeO}_{2}$ and PANI. However, incorporating $\mathrm{CeO}_{2}$ in the polymer decreases flexibility and increases the overall electrical resistance, which may limit the range of applications. Lin et al. [35] fabricated electrospun PANI nanofibers and introduced hydrophilic poly(ethylene oxide) and hydrophobic poly(vinyl butyral) into PANI to tune the sensitivity towards humidity. It was observed that increasing the fraction of the hydrophilic material within the sensor decreased resistance, whereas increasing the hydrophobicity resulted in higher resistance.
Our work here demonstrates the advantages of fabricating PANI nanotubes in combination with a hydrophilic material, namely poly(2-hydroxyethyl methacrylate) (pHEMA), enabling PANI to be used in humidity sensors with higher humidity sensitivity due to the open-mouth structure and the high surface area of the nanotubes. Furthermore, fabricating conductive nanotubes using templates with mesoscopic pores resulted in the alignment of polymer chains parallel to the tube axis, increasing conductivity above that of nonaligned films [36]. In this study, the fabrication of PANI nanotubes and PANI/pHEMA coaxial nanotubes were done via oxidative chemical vapor deposition (oCVD) and initiated chemical vapor deposition (iCVD) to enhance the control and sensitivity level of humidity sensors. By using the vapor deposition method oCVD, we achieved conformal coatings of PANI, which allowed us to produce nanotubes with high purity and controlled wall thickness. Furthermore, the oxidation state of PANI could be controlled by varying the oxidant flowrate for the purpose of achieving conductive emeraldine salt. The oCVD technique is based on step-growth polymerization where the polymerization takes place directly on the surface of the substrate. The oxidant, either liquid [37] or solid [38], and the monomers are delivered into the vacuum system simultaneously, initiating the polymerization reaction on the surface. The key advantages of oCVD are good homogeneity, retention of polymer functional groups due to low reaction temperature $\left(25-100{ }^{\circ} \mathrm{C}\right)$, adequate electrical conductivity for a wide range of applications and high-quality conformal CP thin films on various non-planar surfaces [39-41]. In this study, the vapor-phase oCVD and iCVD techniques were used to conformally coat the walls of the pores of anodized aluminium oxide (AAO) track-etch membranes. The ability to control the thickness with high sensitivity using these vapor phase techniques allowed to produce coaxial nanotubes. The response of these nanotubes to the changes in humidity could be tuned by introducing the hydrogel inner layer.

\section{Results and Discussion}

The deposition of PANI films on a Si wafer was confirmed by Fourier-transform infrared (FTIR) analysis (Figure 1a). The broad peaks at $2850-3100 \mathrm{~cm}^{-1}$ and $3100-3600 \mathrm{~cm}^{-1}$ correspond to $\mathrm{C}-\mathrm{H}$ and $\mathrm{N}-\mathrm{H}$ stretching vibrations, respectively. The peak at $1590 \mathrm{~cm}^{-1}$ can be attributed to the quinoid ring stretching, while the peak at $1495 \mathrm{~cm}^{-1}$ is due to the benzenoid ring stretching [42].

A complementary structural analysis was performed with Raman spectroscopy (Figure 1b). The peak at $1193 \mathrm{~cm}^{-1}$ is due to $\mathrm{C}-\mathrm{H}$ vibrations bending in benzoid units. The peaks at 1223 and $1272 \mathrm{~cm}^{-1}$ correspond to the bands related to amine groups. Between 1332 and $1376 \mathrm{~cm}^{-1}$, the vibrations of delocalized polaronic structures can be observed. The peaks at 1458 and 

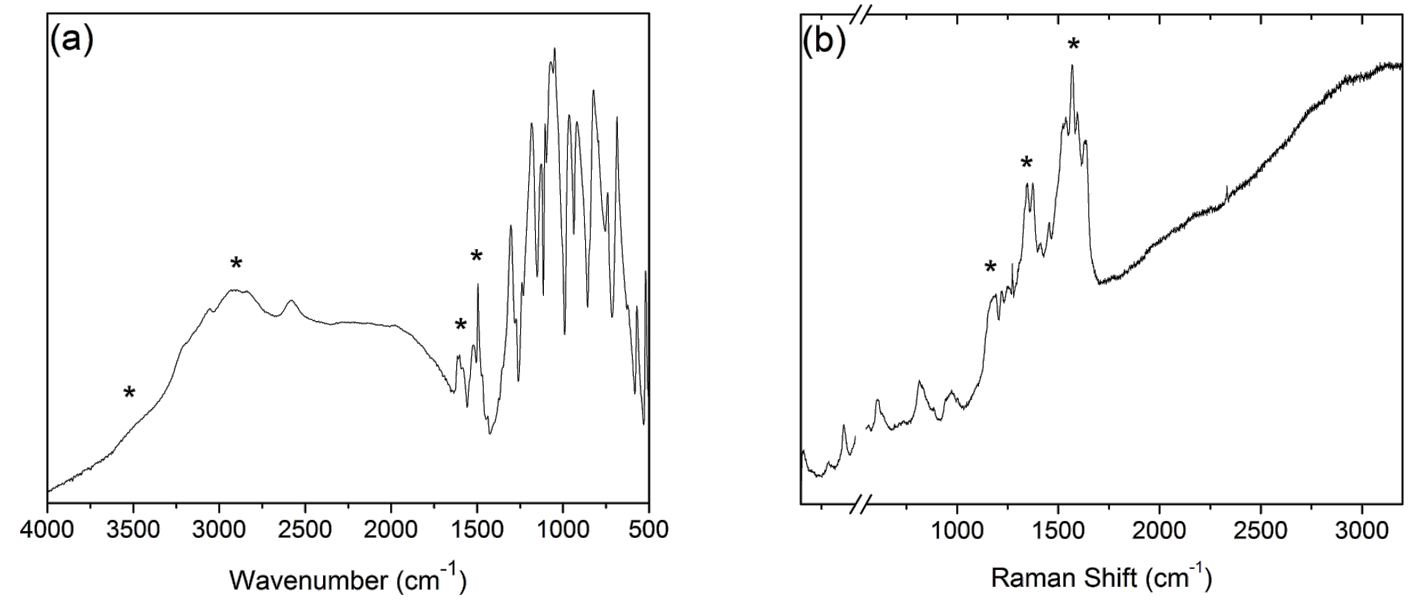

Figure 1: (a) FTIR spectra of PANI emeraldine thin film on a Si wafer. The peaks at 1590 and $1495 \mathrm{~cm}^{-1}$ correspond to stretching vibrations of the quinoid and benzenoid rings, respectively. (b) RAMAN spectra of PANI emeraldine thin film on a Si wafer. The peak at $1638 \mathrm{~cm}^{-1} \mathrm{corresponds} \mathrm{to} \mathrm{the}$ $\mathrm{C}-\mathrm{C}$ stretching vibrations in benzoid units, while the peaks due to the $\mathrm{C}=\mathrm{N}$ and $\mathrm{C}=\mathrm{C}$ stretching vibrations in quinoid units appear at 1458 and $1569 \mathrm{~cm}^{-1}$, respectively. Both the FTIR and RAMAN spectra confirm the polymerization of PANI.

$1569 \mathrm{~cm}^{-1}$ correspond to $\mathrm{C}=\mathrm{N}$ and $\mathrm{C}=\mathrm{C}$ stretching vibrations in quinoid units, respectively. At $1638 \mathrm{~cm}^{-1}$, the peak for $\mathrm{C}-\mathrm{C}$ stretching vibrations in benzoid units is present. The results obtained are in good agreement with literature confirming the successful polymerization of PANI thin films [43].

In order to confirm the formation of the protonated emeraldine salt form of PANI, UV-vis analysis was performed on the thin film samples. Figure 2a shows the UV-vis spectra of the as-deposited PANI films with three characteristic peaks at 360, 430 and $796 \mathrm{~nm}$, indicating the formation of a polaron band transition. Furthermore, the peak at $430 \mathrm{~nm}$ originates from polaron-bipolaron band transitions consistent with the emeraldine salt form of PANI [44].
The band gaps of both annealed $\left(80^{\circ} \mathrm{C}\right)$ and as-deposited PANI samples were found using the UV-vis spectra (Figure $2 b$ ). The band gap of PANI can be calculated from the wavelength of the polaron band excitation [45]. The onset of absorption of the polaron band excitation was used to find the band gap energies, $E_{\mathrm{g}}$, of both samples. The $E_{\mathrm{g}}$ of as-deposited and annealed samples were calculated as 2.38 and $2.26 \mathrm{eV}$, respectively. The slight decrease in the band gap with increasing annealing temperature is consistent with previous PANI studies. Joshi et al. reported that the band gap of PANI decreases as the annealing temperature increases up to $100^{\circ} \mathrm{C}$ because of the formation of a new crystalline region and the rearrangement of the existing crystalline region [46]. However, annealing at temperatures above $100{ }^{\circ} \mathrm{C}$ initiates deformation and causes damage in the
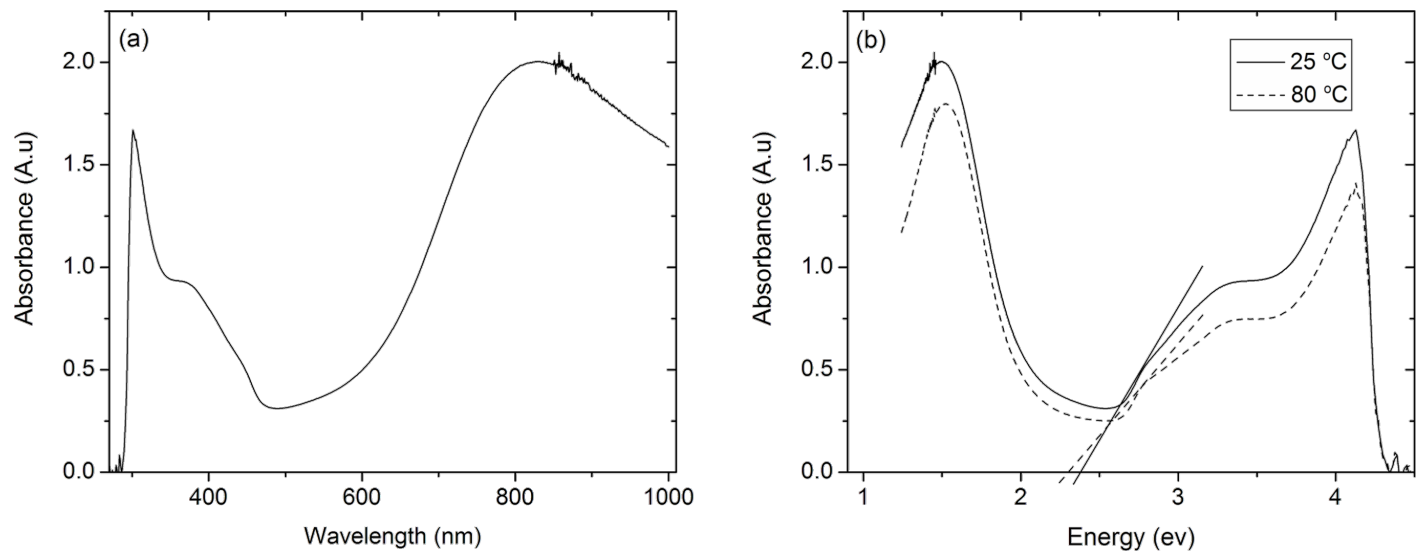

Figure 2: (a) UV-vis spectrum of as-deposited PANI thin films. Three characteristic peaks at 360,430 and $796 \mathrm{~nm}$ indicate the formation of a polaron band transition. The peak at $430 \mathrm{~nm}$ due to the polaron-bipolaron band transition confirms the deposition of the emeraldine salt form of PANI. (b) UV-vis spectra of as-deposited and annealed PANI thin films. Bandgaps of 2.38 and 2.26 eV were calculated for the as-deposited and annealed samples, respectively, confirming the formation of the protonated emeraldine salt form of PANI. 
crystalline structure of PANI polymer chains resulting in the increase of the band gap energy.

The crystallinity of the deposited films was studied by using XRD analysis (Figure 3). The spectra of the non-annealed, as-deposited samples did not show any distinct peaks, indicating the amorphous state of the films. However, after an annealing process at $80^{\circ} \mathrm{C}$ for $4 \mathrm{~h}$, the measurements revealed two peaks at the characteristic $2 \theta$ angles of PANI, $16^{\circ}$ and $25^{\circ}$, which correspond to the (011) and (200) planes, respectively. These results are attributed to the reorganization of the chains during annealing to form crystalline regions. However, the broadness of the peaks indicate a low degree of crystallinity [47].

The surface morphology of the PANI thin films was examined by using AFM analysis (Figure 4). The RMS surface roughness of the as-deposited thin films of $350 \mathrm{~nm}$ thickness was measured as $30 \mathrm{~nm}$ on a flat substrate, and the roughness increased with film thickness. When the annealing temperature was increased, the RMS roughness of the PANI thin films decreased. The decrease in surface roughness with increasing temperatures can be explained by the rearrangement of amorphous part of polymer chains and formation of new crystalline regions on the surface that reduce the irregularity and increase the percentage of crystalline regions on the surface $[48,49]$.

The conductivity studies of the thin film samples were performed with a four-point probe in air. Figure 5 shows the conductivity values of the as-deposited $\left(25^{\circ} \mathrm{C}\right)$ samples and samples annealed at temperatures ranging from 40 to $180{ }^{\circ} \mathrm{C}$ for $4 \mathrm{~h}$. The highest conductivity value of ca. $26 \mathrm{~S} / \mathrm{cm}$ was obtained with the sample annealed at $100{ }^{\circ} \mathrm{C}$. This conductivity increase is attributed to the increase in crystallinity with annealing, which leads to reduced hopping distance between chains and crystal domains [50]. However, above $100{ }^{\circ} \mathrm{C}$ the polymer starts degrading, resulting in damage to the crystalline structure and reduction of the conductivity.

For sensor applications, the long-term stability of the deposited films was investigated. The conductivities of the PANI coated glass were recorded with a four-point probe in air over 30 days. Figure 6 shows that the decrease in the conductivities of the samples was less than $2 \%$ at the end of 30 days, indicating the electrical stability of the films required for long-term applications. Furthermore, the electrical stability of the samples was observed to be independent of the annealing temperatures.

For humidity experiments, circular gold electrodes were evaporated on the PANI films and the resistance was measured using a two-point probe to ensure consistency with the electrical characterization of the nanotube samples. The diameters of the gold electrodes were optimized at $200 \mu \mathrm{m}$ in order to provide better DC resistance. The lower resistance of $3772 \mathrm{k} \Omega$ of PANIcoated glass with electrodes compared to the resistance of $65664 \mathrm{k} \Omega$ of PANI-coated glass without electrodes measured in air can be explained by the reduced contact resistance between the PANI thin film and the probes in the presence of gold electrodes.

For PANI flat films, the actual resistance values $(R)$ versus relative humidity ( $\mathrm{RH} \%$ ) are plotted in Figure 7. The resistance slightly decreases as $\mathrm{RH} \%$ increases up to a certain value (RH\% of $84.3 \%$ ). Above $84.3 \%$ resistance starts to increase with humidity. The change in the conductivity of the PANI polymer with humidity is the result of the increasing doping level of the polymer due to the proton exchange facilitated by the H-bonds between the water molecules and $\mathrm{N}$-atoms in the backbone [51]. The ionizable water molecules dissociate into positive protons and negative hydroxyl ions upon entering the polymer chain.
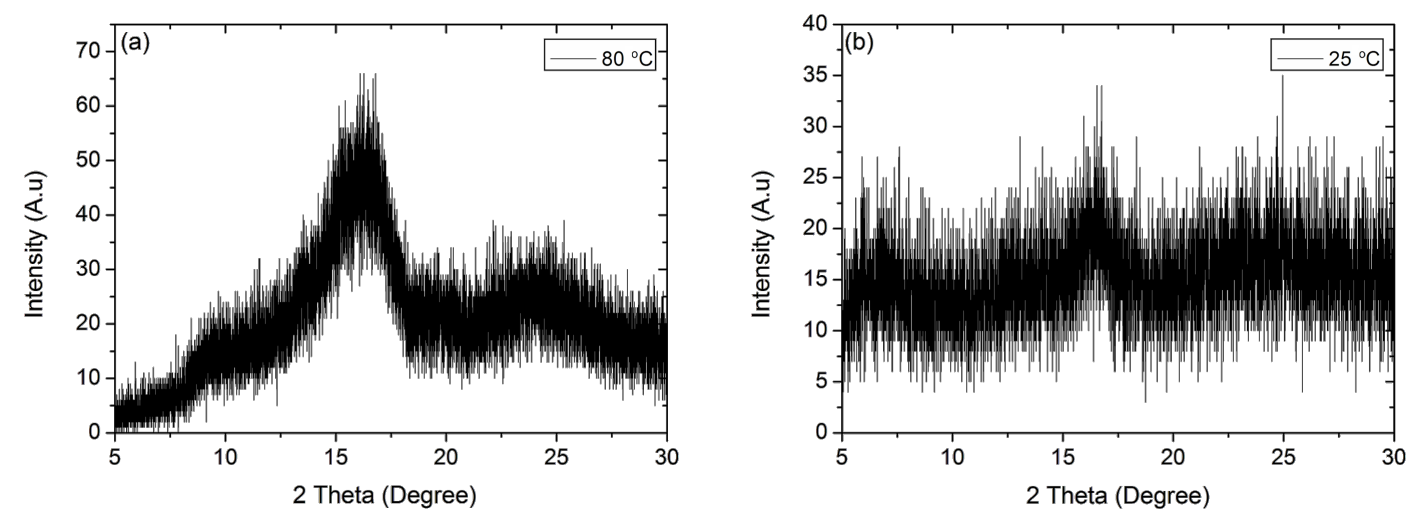

Figure 3: XRD spectra of (a) annealed and (b) as-deposited PANI thin films. After annealing at $80{ }^{\circ} \mathrm{C}$ for $4 \mathrm{~h}$, characteristic peaks of PANI appear at $16^{\circ}$ and $25^{\circ}$, which correspond to (011) and (200) planes, respectively, confirming the crystallinization of the polymer film upon annealing. 


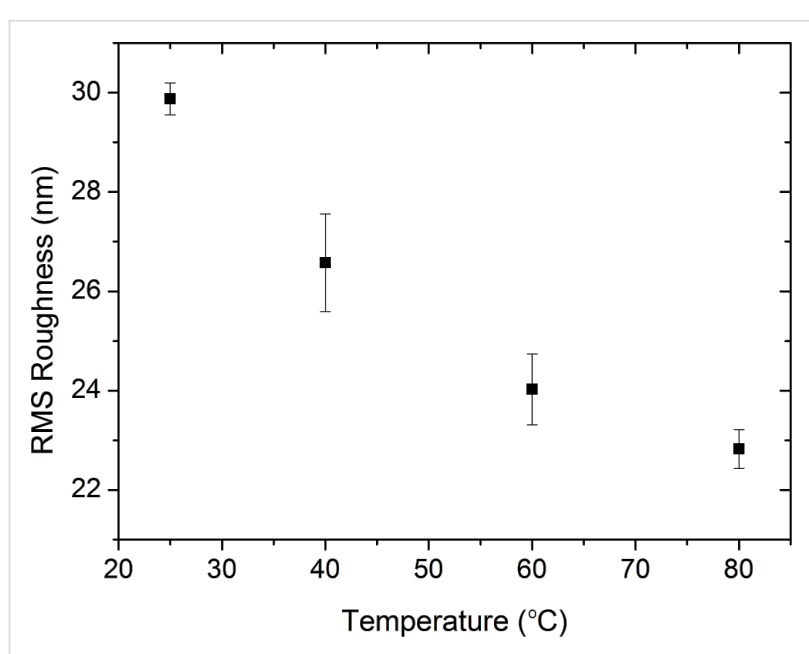

Figure 4: Surface roughness of PANI thin films annealed at 25, 40, 60 and $80^{\circ} \mathrm{C}$. The surface roughness of the polymer thin films decreases as a result of the increased crystallinity as the annealing temperature increases.

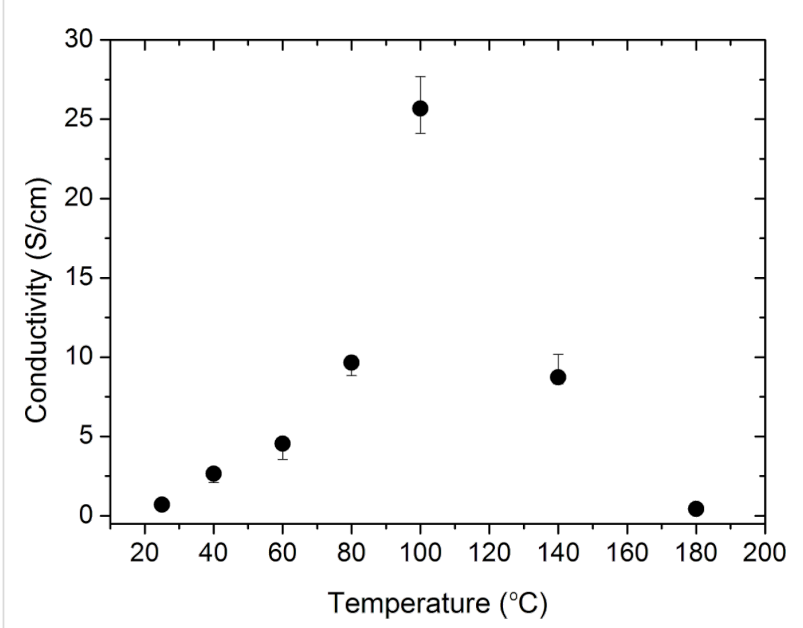

Figure 5: Electrical conductivity of PANI thin films at different annealing temperatures. The conductivity of the films increases with increasing annealing temperatures up to $100^{\circ} \mathrm{C}$ because of the increase in crystallinity. Above $100{ }^{\circ} \mathrm{C}$ the crystalline structure is damaged leading to the reduction of conductivity.

The protons dope the polymer further until the undoped parts of the emeraldine salt is mostly doped with the $\mathrm{H}^{+}$ions, after which swelling starts dominating. Swelling of the polymer due to excess water in the ambient results in higher hoping distances and creates distortion in the polymer chains, reducing the conductivity. The maximum resistance of $4372 \mathrm{k} \Omega$ was obtained at $\mathrm{RH} \%$ of $11.3 \%$, whereas the minimum was obtained at $84.3 \%$ which is $3301 \mathrm{k} \Omega$.

Single-component PANI and coaxial PANI/pHEMA nanotubes were fabricated using sacrificial AAO membranes. The same process parameters during the polymer deposition were used

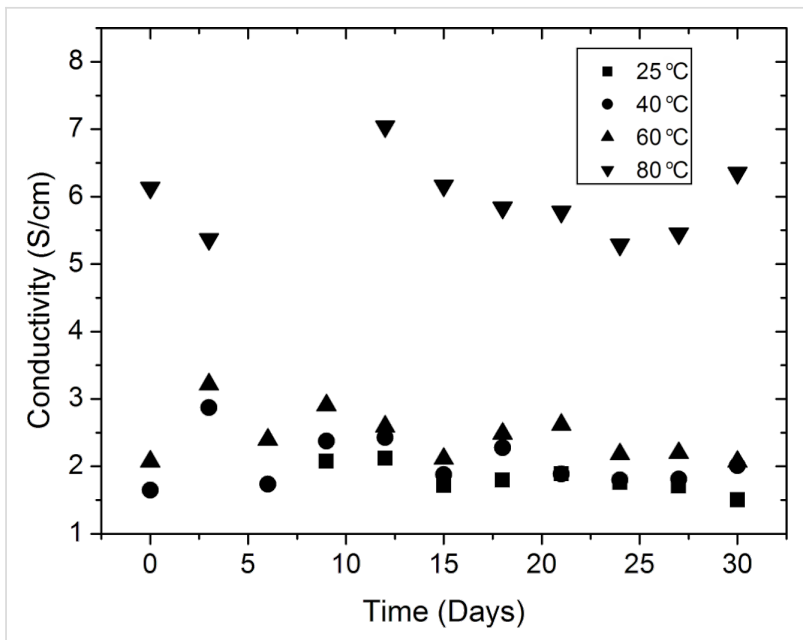

Figure 6: Time dependence of the electrical conductivity of PANI thin films annealed at different temperatures. The decrease in the conductivity after 30 days was less than $2 \%$ and did not depend on the annealing temperature.

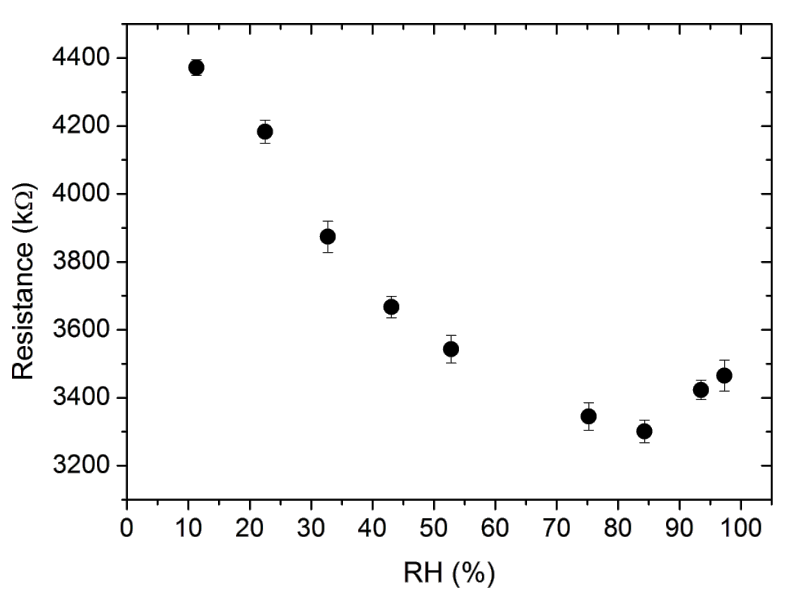

Figure 7: Resistance of PANI thin films as a function of the relative humidity measured using a two-point probe. The parabolic dependency is the result of the competitive effects of doping and swelling of the polymer.

during the fabrication of both nanotubes and thin films. Figure 8 shows the SEM images of the coaxial and single component PANI nanotubes after removal of the AAO membrane. The fabricated nanotubes are approximately $200 \pm 10 \mathrm{~nm}$ in diameter with lengths of 3-4 $\mu \mathrm{m}$.

Circular gold electrodes were evaporated on the nanotubes for the resistance measurements and the change in the resistance of the nanotubes with humidity was measured using two-point probe. For pure PANI nanotubes the resistance at different relative humidity values is shown in Figure 9. The maximum resistance, which is obtained at $97.3 \%$, is $1023 \mathrm{k} \Omega$ while the minimum resistance was measured as $75 \mathrm{k} \Omega$ at $52.8 \%$. It should 


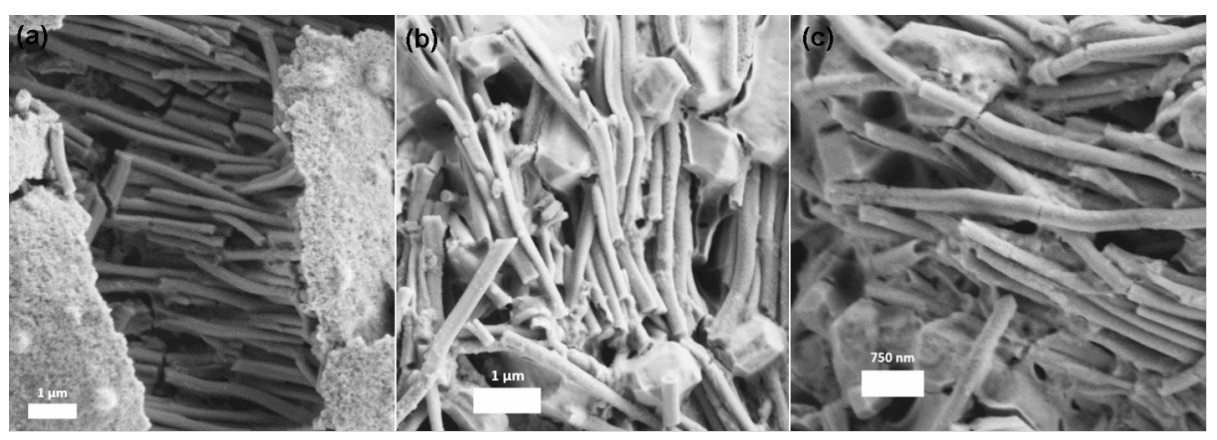

Figure 8: High resolution SEM images of (a) coaxial PANI/pHEMA and (b-c) PANI single component nanotubes.

be noted that since the measurements are taken $30 \mathrm{~s}$ after the samples are removed from the humid environment, the measured resistance values may be smaller than the resistances when the samples are in the humid atmosphere. However, the trend of the resistance change with the humidity is not expected to be affected by this delay.

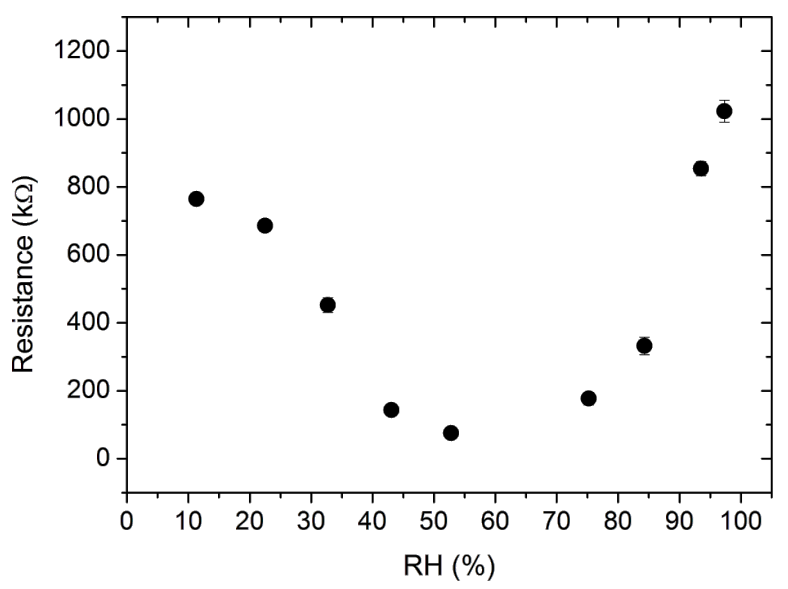

Figure 9: Resistance of single-component PANI nanotubes as a function of the relative humidity. The competing effects of doping and swelling lead to the parabolic behavior, which is similar to the observed behavior in PANI thin films.

The significant resistance difference between PANI nanotubes and PANI thin film stems from the alignment of the polymer chains when deposited inside the pores of a template [36]. This supermolecular order leads to improved conjugation lengths with fewer bends and kinks in the linear polymer chains [52] leading to increased conductivities in nanotubes compared to thin films.

A similar parabolic dependence of resistance on the humidity as observed in the thin films was also observed in the nanotube samples (Figure 9). This type of parabolic dependence of resistance on the humidity was previously reported for PANI nanotubes [53]. The competition between the doping and swelling effects determines at which $\mathrm{RH} \%$ value the reversal in the behavior of resistance will occur [33]. Comparing the $\mathrm{RH} \%$ value at which this reversal occurs for the PANI thin films to that of the nanotubes, it is observed that for PANI nanotubes this transition occurs at $52.8 \%$. This is significantly lower than $84.3 \%$ that is observed for thin films. This difference can be explained by the high surface-to-volume ratios of the nanotubes compared to the thin films. Due to their high surface-to-volume ratios, more polymer chains are exposed to water molecules in the nanotubes. Even lower humidity levels enable the undoped regions of polymer chains of the nanotubes to be doped with $\mathrm{H}^{+}$ions, resulting in an early onset of this transition behavior.

Comparing PANI thin films (Figure 7) to the nanotubes (Figure 9) in terms of the dependence of their resistance on relative humidity, it is observed that the dependence of resistance on $\mathrm{RH} \%$ is significantly stronger for nanotubes compared to the thin films. The resistance of nanotubes changes from $1023 \mathrm{k} \Omega$ to $75 \mathrm{k} \Omega$ whereas for the thin films, the change of resistance is only $25 \%$. The higher sensitivity of the nanotubes to the changes in relative humidity can again be explained by the high surface-to-volume ratio of the nanotubes, enabling more water molecules to interact with polymer chains. This improved sensitivity is especially desirable for humidity sensors.

The main issue with the nanotubes sensor is that within the measurement range of $0-100 \%$ relative humidity, the resistance values go through a minimum, resulting in same resistance values at two different humidity levels [33]. As explained in the earlier sections, this minimum is due to the competition between the swelling and doping effects. Incorporating another polymer layer that is sensitive purely to humidity is expected to distinguish between low and high humidity levels, causing differences in the measured resistance values. For this purpose, 
coaxial nanotubes with pHEMA inner layers and PANI outer layers were fabricated. pHEMA is an insulating hydrogel that is highly sensitive to ambient humidity. The effect of relative humidity on the resistance changes for the coaxial nanotubes is plotted in Figure 10a. According to these results, the resistance values increase parabolically with increasing humidity levels. The increase of resistance is limited (5\%) between the humidity levels of $11.3 \%$ and $32.7 \%$, indicating poor sensitivity, which is not desirable for a sensor. However, above $32.7 \%$ resistance values increase significantly until $97.3 \%$ of relative humidity. The maximum resistance of $4027 \mathrm{k} \Omega$ was obtained at $97.3 \%$, whereas the minimum resistance was $957 \mathrm{k} \Omega$ at the humidity level of $22.5 \%$.

Figure $10 \mathrm{~b}$ compares coaxial nanotubes to the single-component nanotubes in terms of the dependence of their resistance on relative humidity. The significant difference between the singlecomponent and the coaxial nanotubes in terms of the resistance dependence on the humidity levels stems from the existence of hydrogel pHEMA in the coaxial nanotubes. As stated earlier, pHEMA is sensitive to humidity and swells or shrinks in response to the water level in the ambient. As humidity increases, pHEMA swells which results in an increased distance between each polymer chain, affecting the electronic structure of the nanotubes. The swelling of the inner pHEMA layer, leads to an overall increase in the nanotube diameter, and thus swelling of the outer PANI layer. The longer distances between PANI chains increase the hoping resistance of polymer structure so that the resistance of coaxial nanotubes increases with increasing humidity levels. The doping effect, therefore, is dominated by the swelling effect in the presence of pHEMA layer. At low humidity levels (up to $22.5 \%$ ), on the other hand, due to the limited swelling of the pHEMA layer, doping effect balances out the swelling effect, resulting in weaker dependence of resistance on humidity.

The stability of single-component and coaxial PANI nanotubes sensors was studied by performing cyclic measurements at two different $\mathrm{RH} \%$ values. Figure 11a shows the measured resistance of the single-component nanotubes at $\mathrm{RH} \%$ values of $35 \%$ and $52.8 \%$. In the first cycle, the resistance was measured as $336 \mathrm{k} \Omega$ and $79 \mathrm{k} \Omega$ at $35 \%$ and $52.8 \%$, respectively, whereas, in the tenth cycle, the PANI resistance was $334 \mathrm{k} \Omega$ and $87 \mathrm{k} \Omega$ at $35 \%$ and $52.8 \%$, respectively. The change in measured resistance values at both humidity levels is lower than $10 \%$, indicating the stability of the sensors at the end of 10 cycles. The cyclic measurements of coaxial nanotubes were repeated 10 times at RH\% values of (35\%) and 22.5\% (Figure 11(b)). In the first cycle, the resistance was measured as $1154 \mathrm{k} \Omega$ and $943 \mathrm{k} \Omega$ at $35 \%$ and $22.5 \%$, respectively. In the tenth cycle, the resistance was $1246 \mathrm{k} \Omega$ and $983 \mathrm{k} \Omega$, at $35 \%$ and $22.5 \%$, respectively. The change in the resistance values at the end of 10 cycles is less than $10 \%$, confirming the stability of the nanotubes.

\section{Conclusion}

Single-component conducting PANI nanotubes and coaxial polymer nanotubes with PANI outer layer and hydrogel pHEMA inner layer were synthesized using iCVD and oCVD techniques. Ability to control the thickness during deposition via these vapor-phase methods allowed depositing two layers of different polymers inside the pores of AAO track-etch membranes.

The characterization of the PANI thin films deposited by the vapor-phase oCVD method showed that crystalline PANI thin
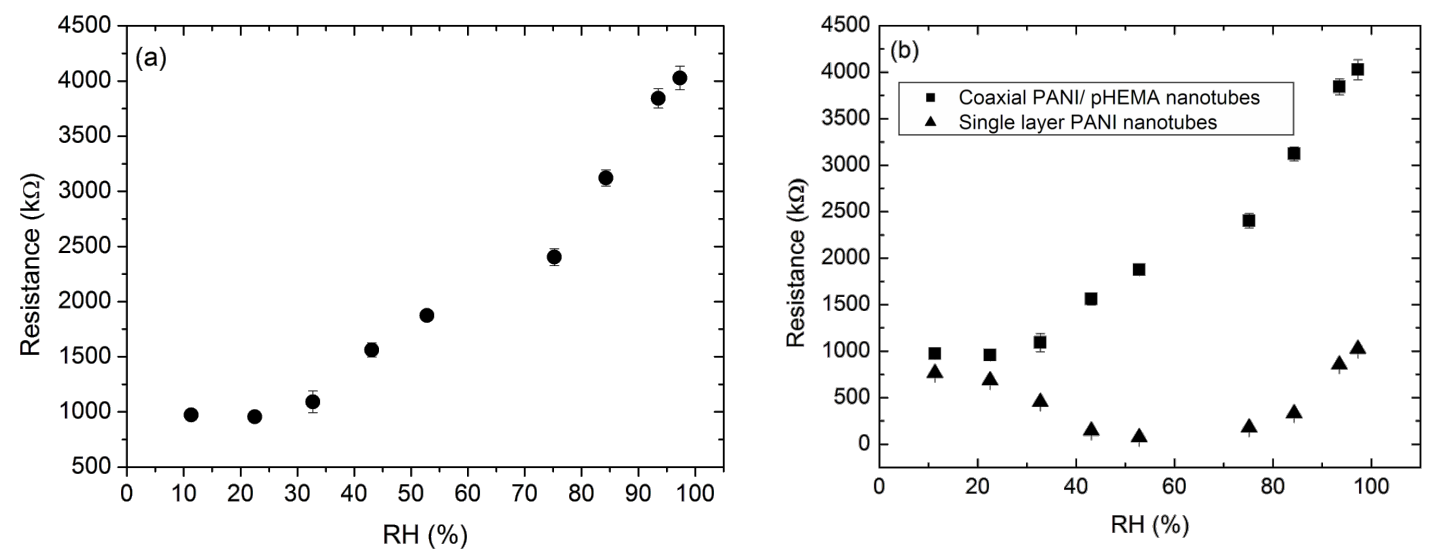

Figure 10: (a) Change in the resistance of coaxial PANI/pHEMA nanotubes with relative humidity. (b) Comparison of the single component and coaxial nanotubes in terms of humidity effect on resistance. The parabolic behavior observed in single-component nanotubes is not observed in the coaxial nanotubes, suggesting the dominance of the swelling effect in the presence of pHEMA hydrogel inner layer. 

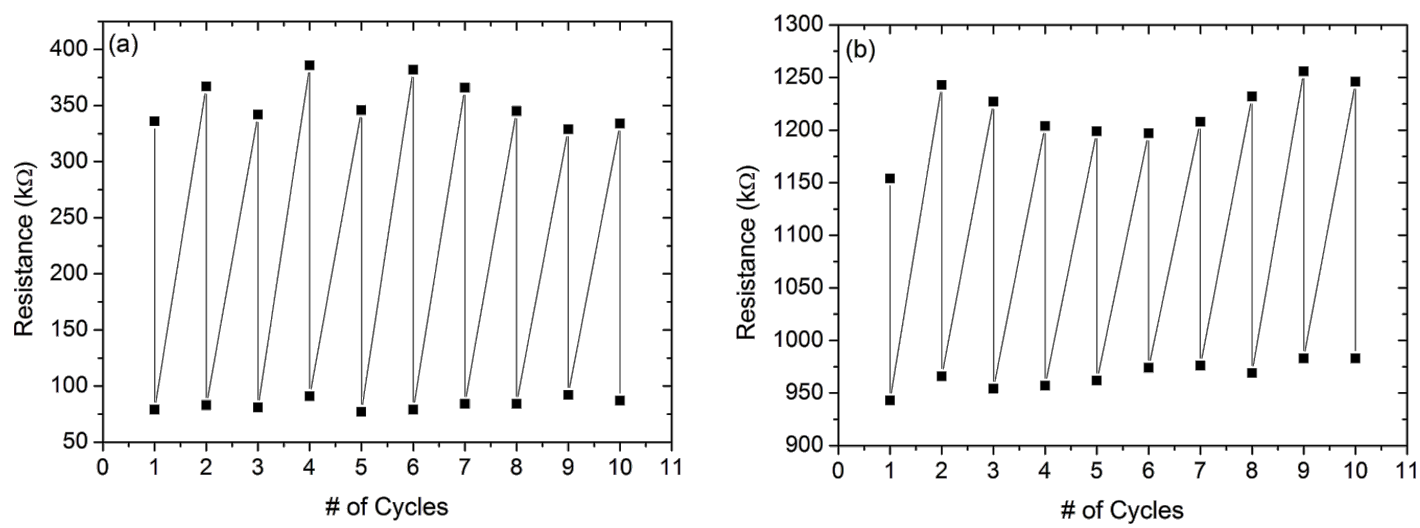

Figure 11: Cyclic resistance measurements of (a) the single component PANI nanotubes at RH $\%$ of $35 \%$ and $52.8 \%$ and (b) coaxial PANI/pHEMA nanotubes at $\mathrm{RH} \%$ of $35 \%$ and $22.5 \%$. At the end of 10 cycles, the change in the resistance is less than $10 \%$.

films could be obtained upon annealing the samples. Conductivity also increased with annealing temperature due to the reduced hopping distance between chains and crystal domains. The results obtained agree with the conventional, solution-based PANI deposition methods reported in literature.

Performance of the single-component and coaxial nanotubes as humidity sensors were tested under different humidity conditions. The competition between the doping and swelling mechanisms of the polymer upon exposure to humidity leads to an increase in the conductivity with humidity until a specific humidity level, above which conductivity decreases. To improve the sensitivity of the sensors an inner hydrogel layer was introduced, delaying the response of the PANI layer. The hydrogel layer in the coaxial nanotubes might further facilitate the swelling effect, which dominates doping, resulting in resistance values that continuously increase with humidity.

The ability to tune the response of the nanotube sensors to humidity by introducing a hydrogel layer will help to improve the sensitivity of the sensors. Furthermore, by incorporating different polymers in the coaxial sensors application areas of these sensors can be extended.

\section{Experimental}

The monomers aniline (99.5\%, Sigma-Aldrich), HEMA (99\%, Sigma Aldrich), the crosslinker ethylene glycol dimethacrylate (98\%, Sigma Aldrich) (EGDMA), the initiator tert-butyl peroxide (98\%, Sigma Aldrich) (TBPO) and the oxidant antimony pentachloride (99\%, Sigma Aldrich) $\left(\mathrm{SbCl}_{5}\right)$ were used as received.

Aniline was heated in a metal jar up to $60{ }^{\circ} \mathrm{C}$ while $\mathrm{SbCl}_{5}$ was kept at room temperature in a glass jar. Both chemicals were delivered to the system in vapor phase through different ports facing the substrate surface. Glass slides, $\mathrm{Si}(100)$ wafers and anodic aluminum oxide template (AAO) with pore sizes of $200 \mathrm{~nm}$ were used as substrates and were coated simultaneously. Glass slides were used for electrical conductivity measurements and UV-visible spectroscopy (UV-vis). Si wafers were used for Fourier-transform infrared (FTIR) spectrophotometry, Raman spectroscopy, atomic force microscopy (AFM) and electron microscopy. AAO templates were used for PANI nanotube synthesis. During deposition, the flowrates of aniline and $\mathrm{SbCl}_{5}$ were maintained at $1.6 \mathrm{sccm}$ and $1.2 \mathrm{sccm}$, respectively, at $25 \mathrm{mTorr}$ of operating pressure for $15 \mathrm{~min}$. The stage temperatures varied between 25 and $80{ }^{\circ} \mathrm{C}$ for different experiments. After the polymer coating, PANI-coated Si wafers were annealed at temperatures ranging between 40 and $180{ }^{\circ} \mathrm{C}$ for $4 \mathrm{~h}$ in a vacuum oven.

For PANI flat thin film characterization, FTIR spectrophotometry (Thermo Fischer Scientific Model NICOLETiS10) and Raman spectrometry (Renishaw, inVia Reflex) were used to analyze chemical properties of PANI. FTIR spectra were acquired with $4 \mathrm{~cm}^{-1}$ resolution. Raman measurements were carried out at $532 \mathrm{~nm}$ wavelength and $50 \mathrm{~mW}$ power. Furthermore, UV-vis spectrometry (Shimadzu, UV-VIS 3150) was used on PANI-coated glass slides in order to find the band gap of fabricated PANI and to confirm the electrical conductivity of the film by calculating band-transition energies. Atomic force microscopy (Bruker Multimode 8, ScanAsyst) was used to acquire topography and surface roughness of as-deposited and annealed PANI samples.

X-ray diffraction (XRD) (Bruker, D8 Advance XRD) analysis was performed to study the crystalline state of the annealed and as-deposited PANI films. The measurements were taken on as-deposited PANI and PANI samples annealed at $80{ }^{\circ} \mathrm{C}$ at $2 \theta$ angles of $5-40^{\circ}$ in order to eliminate the peak originating 
from the Si(100) planes. Each measurement took $4 \mathrm{~h}$ for an adequate signal-to-noise ratio. Thickness of the films on Si wafer and glass substrates was measured with a spectroscopic ellipsometer (M-2000, J. A. Woollam) at 65,70 , and $75^{\circ}$ within a range of 300-800 $\mathrm{nm}$. For electrical characterization of the PANI thin films, $I-V$ curves were obtained using a fourpoint probe at $0.01 \mu \mathrm{A}$ and the measurements were taken at four different locations (Lucas Labs Pro 4, Keithley 2400 Sourcemeter).

For the fabrication of the coaxial PANI/pHEMA nanotubes PANI was first deposited on AAO templates via oCVD and then the coated templates were exposed to oxygen plasma to remove excess polymer layer on top of AAO membranes. Subsequently, PANI-coated templates were put inside an iCVD chamber for pHEMA deposition. During iCVD depositions, HEMA and EGDMA were heated up to 70 and $85^{\circ} \mathrm{C}$, respectively, and TBPO was kept at room temperature. The deposition was performed at $120 \mathrm{mTorr}$ with a stage temperature of $40{ }^{\circ} \mathrm{C}$. The flowrates of HEMA, EGDMA and TBPO were set to $0.8,0.11$ and $1 \mathrm{sccm}$, respectively. After the deposition, AAO templates coated with PANI and pHEMA were exposed to oxygen plasma (Torr) at $50 \mathrm{~W}$ to remove the excess film on the top of the templates. Afterwards, AAO templates were attached to Si wafers and immersed in $0.5 \mathrm{M} \mathrm{HCl}$ solution for $48 \mathrm{~h}$ to release the coaxial nanotubes, which were then allowed to dry in the air for two days. This allowed immobilization of the freestanding nanotubes on Si wafers for imaging and sensor studies. Figure 12 shows the fabrication steps of nanostructures used in this study. The synthesized coaxial nanotubes had PANI on the outer side and pHEMA inside. Images of the nanotubes were taken with a field-emission scanning electron microscope (FESEM, Zeiss, SUPRA VP 35).

For the electrical characterization of the PANI thin films and PANI/pHEMA nanotubes, an array of chrome $(3 \mathrm{~nm})$ and gold $(150 \mathrm{~nm})$ electrodes with a diameter of $200 \mu \mathrm{m}$ and a spacing of $200 \mu \mathrm{m}$ were deposited on the nanotubes and thin films using an e-beam evaporator (Torr). Prior to the e-beam evaporation, conventional lithography with a shadow mask was used to create a pattern for the electrodes. The photoresist AZ 5214 E

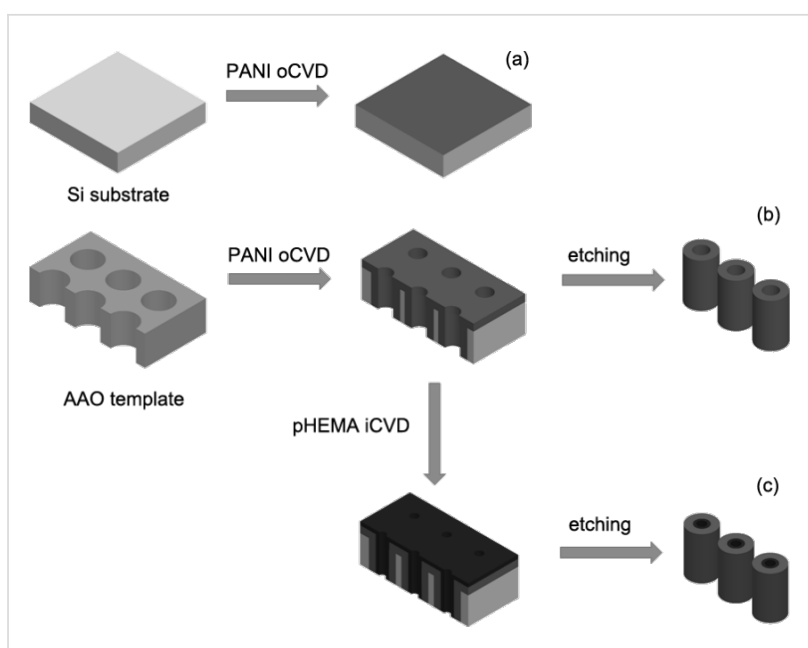

Figure 12: Fabrication steps of nanostructures. (a) PANI thin films are prepared by coating thin layer of PANI on Si surfaces using oCVD. (b) Single-component PANI nanotubes are fabricated by coating the pores of templates with PANI using oCVD. The templates are then etched to release the nanotubes. (c) Coaxial PANI/pHEMA nanotubes are prepared by first coating the pores of templates with PANI polymer using oCVD, followed by iCVD coating of the pores with pHEMA. As the final step the templates are etched to release the nanotubes.

(Merck GmbH), the developer AZ 726 (MIF) (Merck GmbH) and deionized water (stopper) were used for the lithography. After the e-beam evaporation, samples were annealed at $100{ }^{\circ} \mathrm{C}$ for $4 \mathrm{~h}$. Optical microscope (Zeiss, Axio Scope A1 MAT) image was taken to show the gold electrodes on the PANI thin film surface.

Both thin film and nanotube samples were then tested for sensor performance. For this purpose, a 2-Point probe (Keithley, 2401 Sourcemeter) was used for resistance measurements with constant DC current of $1 \mu \mathrm{A}$. In order to vary ambient humidity, several saturated salt solutions with different humidity levels were prepared in DI water. Table 1 lists the salt solutions and their relative humidity $(\mathrm{RH})$ at room temperature.

Each salt solution and the samples were placed in a sealed box to isolate them from air (Figure 13) For resistance measurements, the samples were taken out from the sealed box and put on the two-point probe station. The initial measurements were

Table 1: Salt solutions and the relative humidity levels obtained in a sealed box by using these solutions.

\begin{tabular}{ccccc} 
salt solution & $\mathrm{LiCl}$ & $\mathrm{CH}_{3} \mathrm{CO}_{2} \mathrm{~K}$ & $\mathrm{MgCl}_{2}$ & $\mathrm{~K}_{2} \mathrm{CO}_{3}$ \\
\hline relative humidity & $11.3 \%$ & $22.5 \%$ & $32.7 \%$ & $43.1 \%$ \\
\hline salt solution & $\mathrm{NaCl}$ & $\mathrm{KCl}$ & $\mathrm{KNO}_{3}$ & $\mathrm{~K}_{2} \mathrm{SO}_{4}$ \\
\hline relative humidity & $75.2 \%$ & $84.3 \%$ & $93.5 \%$ & $97.3 \%$
\end{tabular}


(a)

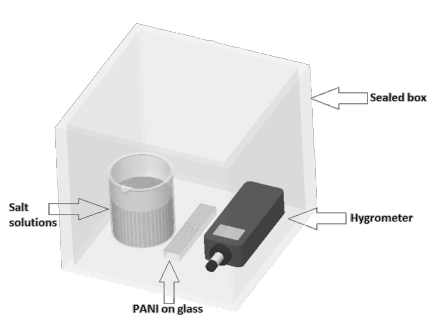

(b)
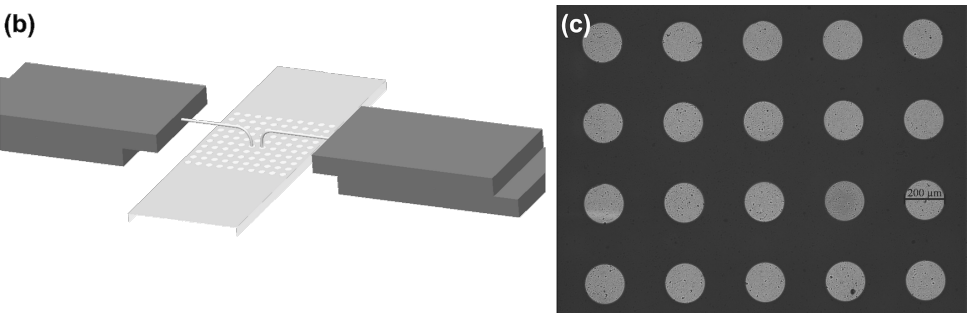

Figure 13: Experimental setup of humidity sensor measurements. (a) The sealed box containing the salt solution, the hygrometer and the nanotube sample. (b) The two-point probe station used for the resistance measurements. The probe station is outside the sealed box. (c) Optical microscope image of the gold electrodes.

taken $30 \mathrm{~s}$ after removing the samples from the sealed box. The cyclic resistance measurements were done by leaving the sample in ambient air $(35 \% \mathrm{RH})$ for 10 min then measuring the resistance of the sample and repeating the same process for $52.8 \%$ RH environment within a sealed box.

\section{Acknowledgements}

This work was supported by the TUBITAK (The Scientific and Technological Research Council of Turkey) Support Program for Scientific and Technological Research Projects (Grant 114M165) and TUBA (Turkish Academy of Sciences) Young Scientist Award Program.

\section{References}

1. Gaharwar, A. K.; Peppas, N. A.; Khademhosseini, A. Biotechnol. Bioeng. 2014, 111, 441-453. doi:10.1002/bit.25160

2. Rapoport, N. Prog. Polym. Sci. 2007, 32, 962-990. doi:10.1016/j.progpolymsci.2007.05.009

3. Peteu, S. F.; Oancea, F.; Sicuia, O. A.; Constantinescu, F.; Dinu, S. Polymers (Basel, Switz.) 2010, 2, 229-251. doi:10.3390/polym2030229

4. de Azeredo, H. M. C.; Mattoso, L. H. C.; McHugh, T. H. Nanocomposites in Food Packaging - A Review. In Advances in Diverse Industrial Applications of Nanocomposites; Reddy, B., Ed.; In Tech, 2011; pp 57-78. doi:10.5772/14437

5. Liu, L.; Ye, X.; Wu, K.; Han, R.; Zhou, Z.; Cui, T. Sensors 2009, 9, 1714-1721. doi:10.3390/s90301714

6. Krogstrup, P.; Jørgensen, H. I.; Heiss, M.; Demichel, O.; Holm, J. V.; Aagesen, M.; Nygard, J.; Morral, A. F. Nat. Photonics 2013, 7, 306-310. doi:10.1038/nphoton.2013.32

7. Du, F.-S.; Wang, Y.; Zhang, R.; Li, Z.-C. Soft Matter 2010, 6, 835-848. doi:10.1039/B915020J

8. Chen, J.; Wang, F.; Liu, Q.; Du, J. Chem. Commun. 2014, 50, 14482-14493. doi:10.1039/C4CC03001J

9. Jang, J.; Yoon, H. Langmuir 2005, 21, 11484-11489. doi:10.1021/la051447u

10. Jang, J.; Chang, M.; Yoon, H. Adv. Mater. 2005, 17, 1616-1620. doi:10.1002/adma.200401909

11. Li, G.; Zhang, Z. Macromolecules 2004, 37, 2683-2685. doi:10.1021/ma035891k

12. Koo, Y. K.; Kim, B. H.; Park, D. H.; Joo, J. Mol. Cryst. Liq. Cryst. 2004, 425, 55-60. doi:10.1080/15421400490506496
13. Higuchi, T.; Nishiyama, H.; Suga, M.; Watanabe, H.; Takahara, A.; Jinnai, H. Microscopy (Oxford, U. K.) 2015, 64, 205-212. doi:10.1093/jmicro/dfv013

14. Huang, J.; Wang, K.; Wei, Z. J. Mater. Chem. 2010, 20, 1117-1121. doi:10.1039/B919928D

15. Boroumand, F. A.; Fry, P. W.; Lidzey, D. G. Nano Lett. 2005, 5, 67-71. doi:10.1021/nl048382k

16. Habisreutinger, S. N.; Leijtens, T.; Eperon, G. E.; Stranks, S. D.; Nicoholas, R. J.; Snaith, H. J. Nano Lett. 2014, 14, 5561-5568. doi:10.1021/nl501982b

17. Peng, S.; Zhu, P.; Wu, Y.; Mhaisalkar, S. G.; Ramakrishna, S. RSC Adv. 2012, 2, 652-657. doi:10.1039/C1RA00618E

18. Sumboja, A.; Tefashe, U. M.; Wittstock, G.; Lee, P. S. Adv. Mater. Interfaces 2015, 2, 1400154. doi:10.1002/admi.201400154

19. Liu, M.-C.; Dai, C.-L.; Chan, C.-H.; Wu, C.-C. Sensors 2009, 9 , 869-880. doi:10.3390/s90200869

20. Abidian, M. R.; Kim, D.-H.; Martin, D. C. Adv. Mater. 2006, 18, 405-409. doi:10.1002/adma.200501726

21. Mohammedi, A.; Hassan, M.-A.; Liedberg, B.; Lundstrom, I.; Salaneck, W. R. Synth. Met. 1986, 14, 189-197. doi:10.1016/0379-6779(86)90183-9

22. Winther-Jensen, B.; Chen, J.; West, K.; Wallace, G. Macromolecules 2004, 37, 5930-5935. doi:10.1021/ma049365k

23. Virji, S.; Huang, J.; Kaner, R. B.; Weiller, B. H. Nano Lett. 2004, 4, 491-496. doi:10.1021/nI035122e

24. Takhira, P. Y.; Versimaha, Y. I.; Aksimentyeva, O. I.; Cizh, B. R.; Cherpak, V. V. Phys. Chem. Solid State 2005, 6, 96-98.

25. Anderson, M. R.; Mattes, B. R.; Reiss, H.; Kaner, R. B. Science 1991, 252, 1412-1415. doi:10.1126/science.252.5011.1412

26. Macdiarmid, A. G.; Chiang, J. C.; Richter, A. F.; Epstein, A. J. Synth. Met. 1987, 18, 285-290. doi:10.1016/0379-6779(87)90893-9

27. Gospodinova, N.; Terlemezyan, L. Prog. Polym. Sci. 1998, 23, 1443-1484. doi:10.1016/S0079-6700(98)00008-2

28. Bhadra, S.; Khastgir, D.; Singha, N. K.; Lee, J. H. Prog. Polym. Sci. 2009, 34, 783-810. doi:10.1016/j.progpolymsci.2009.04.003

29. Kulkarni, V. G.; Campbell, L. D.; Mathew, W. R. Synth. Met. 1989, 30, 321-325. doi:10.1016/0379-6779(89)90654-1

30. Yoo, J. E.; Cross, J. L.; Bucholz, T. L.; Lee, K. S.; Espe, M. P.; Loo, Y.-L. J. Mater. Chem. 2007, 17, 1268-1275. doi:10.1039/b618521e

31. Zhang, Y.; Rutledge, G. C. Macromolecules 2012, 45, 4238-4246. doi:10.1021/ma3005982

32. Wang, J.; Chan, S.; Carlson, R. R.; Luo, Y.; Ge, G.; Ries, R. S.; Heath, J. R.; Tseng, H.-R. Nano Lett. 2004, 4, 1693-1697. doi:10.1021/nl049114p 
33. Zeng, F.-W.; Liu, X.-X.; Diamond, D.; Lau, K. T. Sens. Actuators, B 2010, 143, 530-534. doi:10.1016/j.snb.2009.09.050

34. Parvatikar, N.; Jain, S.; Bhoraskar, S. V.; Prasad, M. V. N. A. J. Appl. Polym. Sci. 2006, 102, 5533-5537. doi:10.1002/app.24636

35. Lin, Q.; Li, Y.; Yang, M. Sens. Actuators, B 2012, 161, 967-972. doi:10.1016/j.snb.2011.11.074

36. Cai, Z.; Martin, C. R. J. Am. Chem. Soc. 1989, 111, 4138-4139. doi:10.1021/ja00193a077

37. Chelawat, H.; Vaddiraju, S.; Gleason, K. Chem. Mater. 2010, 22, 2864-2868. doi:10.1021/cm100092c

38. Lee, S.; Gleason, K. K. Adv. Funct. Mater. 2015, 25, 85-93. doi:10.1002/adfm.201402924

39. Vaddiraju, S.; Cebeci, H.; Gleason, K. K.; Wardle, B. L. ACS Appl. Mater. Interfaces 2009, 1, 2565-2572. doi:10.1021/am900487z

40. Baxamusa, S. H.; Im, S. G.; Gleason, K. K. Phys. Chem. Chem. Phys. 2009, 11, 5227-5240. doi:10.1039/b900455f

41. Trujillo, N. J.; Barr, M. C.; Im, S. G.; Gleason, K. K. J. Mater. Chem. 2010, 20, 3968-3972. doi:10.1039/b925736e

42. Ping, Z. J. Chem. Soc., Faraday Trans. 1996, 92, 3063-3067. doi:10.1039/FT9969203063

43. Gomes, E. C.; Oliveira, M. A. S. Am. J. Polym. Sci. 2012, 2, 5-13. doi:10.5923/j.ajps.20120202.02

44. Flavel, B. S.; Yu, J.; Shapter, J. G.; Quinton, J. S. Soft Matter 2009, 5, 164-172. doi:10.1039/B809609K

45. Stafström, S.; Brédas, J. L.; Epstein, A. J.; Woo, H. S.; Tanner, D. B.; Huang, W. S.; MacDiarmid, A. G. Phys. Rev. Lett. 1987, 59, 1464-1467. doi:10.1103/PhysRevLett.59.1464

46. Joshi, G. P.; Saxena, N. S.; Sharma, T. P.; Mishra, S. C. K. Indian J. Pure Appl. Phys. 2006, 44, 786-790.

47. Chaudhari, H. K.; Kelkar, D. S. Polym. Int. 1997, 42, 380-384. doi:10.1002/(SICI)1097-0126(199704)42:4<380::AID-PI727>3.0.CO;2$\mathrm{F}$

48. Motaung, D. E.; Malgas, G. F.; Arendse, C. J.; Mavundla, S. E.; Oliphant, C. J.; Knoesen, D. J. Mater. Sci. 2009, 44, 3192-3197. doi:10.1007/s10853-009-3425-8

49. Chen, J.-T.; Chen, W.-L.; Fan, P.-W.; Yao, I.-C. Macromol. Rapid Commun. 2014, 35, 360-366. doi:10.1002/marc.201300290

50. Varma, S. J.; Xavier, F.; Varghese, S.; Jayalekshmi, S. Polym. Int. 2012, 61, 743-748. doi:10.1002/pi.4131

51. Travers, J. P.; Nechtschein, M. Synth. Met. 1987, 21, 135-141. doi:10.1016/0379-6779(87)90077-4

52. Martin, C. R. Acc. Chem. Res. 1995, 28, 61-68. doi:10.1021/ar00050a002

53. Long, Y.; Chen, Z.; Wang, N.; Ma, Y.; Zhang, Z.; Zhang, L.; Wan, M. Appl. Phys. Lett. 2003, 83, 1863. doi:10.1063/1.1606864

\section{License and Terms}

This is an Open Access article under the terms of the Creative Commons Attribution License

(http://creativecommons.org/licenses/by/4.0), which permits unrestricted use, distribution, and reproduction in any medium, provided the original work is properly cited.

The license is subject to the Beilstein Journal of Nanotechnology terms and conditions:

(http://www.beilstein-journals.org/bjnano)

The definitive version of this article is the electronic one which can be found at:

doi:10.3762/bjnano.8.89 